\title{
Parameterized Tree Systems
}

\author{
Parosh Aziz Abdulla ${ }^{1}$, Noomene Ben Henda ${ }^{1}$, \\ Giorgio Delzanno ${ }^{2}$, Frédéric Haziza ${ }^{1}$, and Ahmed Rezine ${ }^{1}$ \\ 1 Uppsala University, Sweden \\ 2 Università di Genova, Italy \\ parosh@it.uu.se, \\ Noomene.BenHenda@it.uu.se, \\ giorgio@disi.unige.it, \\ Frederic.Haziza@it.uu.se, \\ Rezine.Ahmed@it.uu.se
}

\begin{abstract}
Several recent works have considered parameterized verification, i.e. automatic verification of systems consisting of an arbitrary number of finite-state processes organized in a linear array. The aim of this paper is to extend these works by giving a simple and efficient method to prove safety properties for systems with tree-like architectures. A process in the system is a finite-state automaton and a transition is performed jointly by a process and its parent and children processes. The method derives an over-approximation of the induced transition system, which allows the use of finite trees as symbolic representations of infinite sets of configurations. Compared to traditional methods for parameterized verification of systems with tree topologies, our method does not require the manipulation of tree transducers, hence its simplicity and efficiency. We have implemented a prototype which works well on several nontrivial tree-based protocols.
\end{abstract}

\section{Introduction}

In recent years, there has been an extensive amount of work on the verification of parameterized systems, e.g. $[11,18,5,9,10]$. Typically, a parameterized system consists of an arbitrary number of finite-state processes organized in a linear array. The task is to perform parameterized verification, i.e. to verify correctness of the system regardless of the number of processes inside the system. Examples of parameterized systems include mutual exclusion algorithms, bus protocols, telecommunication protocols, multi-threaded programs, and cache coherence protocols. This work aims at extending the paradigm of parameterized verification in order to verify systems which operate on tree-like architectures. More precisely, we consider analysis of safety properties for parameterized tree systems. Such a system consists of an arbitrary number of finite-state processes which operate on a tree-like architecture. Examples of parameterized tree systems include several interesting protocols such as the percolate protocol [18],the Tree-arbiter protocol [8], and the IEEE 1394 Tree identity protocol [17].

One of the most prominent techniques which have been used for verification of parameterized tree systems is that of tree regular model checking $[14,4,18,12,7]$.

K. Suzuki et al. (Eds.): FORTE 2008, LNCS 5048, pp. $69-83,2008$.

(C) IFIP International Federation for Information Processing 2008 
In tree regular model checking, configurations (states) of the system are represented by trees, sets of configurations by tree automata, and transitions by tree automata operating on pairs of trees, i.e. tree transducers. Safety properties can be checked through performing reachability analysis, which amounts to applying the tree transducer relation iteratively to the set of initial configurations. The main problem with transducer-based techniques, such as the ones mentioned above, is that they are very heavy and usually rely on several layers of computationally expensive automata-theoretic constructions; in many cases severely limiting their applicability.

In this paper, we propose a light-weight approach to parameterized tree verification which, in addition to its simplicity, also yields a much more efficient implementation than tree regular model checking. In our method, a configuration of the system is represented by a tree over a finite alphabet, where elements of the alphabet represent the local states of the individual processes. The behaviour of the system is induced by a set of rewriting rules which describe how the processes perform transitions. A transition performed by a process is conditioned by the current local state of the process and possibly the local states of neighboring processes, i.e. the parent and children processes. The transition may change the states of all involved processes. (see Figure 1).

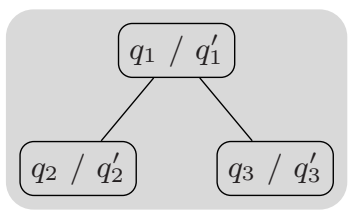

Fig. 1. A typical transition rule where a process and its two children change state from $q_{1}, q_{2}, q_{3}$ to $q_{1}^{\prime}, q_{2}^{\prime}, q_{3}^{\prime}$, respectively

Observe that the set of configurations is infinite since we are dealing with trees of an arbitrary size. In fact, parameterized verification amounts to analyzing an infinite family of systems; namely one for each size of the system and one for each tree of that particular size.

The main idea of our method is to consider a transition relation which is an over-approximation of the one induced by the tree parameterized system. To do so, we modify the semantics of the transition rules, such that a rule is applied to a node and two nodes in its left and right subtrees (rather than its left and right children). The approximate transition system obtained in this manner is monotonic with respect to the tree embedding relation on configurations (larger configurations are able to simulate smaller ones). Since the approximate transition relation is monotonic, it can be analyzed using symbolic backward reachability algorithm based on a generic method introduced in [2]. An attractive feature of this algorithm is that it operates on sets of configurations which are upward closed with respect to the tree embedding relation. This allows an 
efficient symbolic representation of upward sets of configurations, since such a set can be represented by (the finite set of) its minimal elements. Since the minimal elements are trees, reachability analysis can be performed by computing predecessors of trees, which is much simpler and more efficient than applying transducer relations on general tree regular languages. Also, as a side effect, the analysis of the approximate model is guaranteed to terminate. This follows from the fact that the embedding relation on configurations (trees) is a well quasi-ordering by Kruskal's theorem [19]. The whole verification process is fully automatic since both the approximation and the reachability analysis are carried out without user intervention. Observe that if the approximate transition system satisfies a safety property then we can safely conclude that the original system satisfies the property too.

Based on the method, we have implemented a prototype which works well on several tree-based protocols such as the percolate, leader election, Tree-arbiter, and the IEEE 1394 Tree identity protocols.

Outline. In the next section, we give some preliminaries on trees. In Section 3, we define the basic model of parameterized tree systems. In Section 4, we describe the induced transition system and in Section 5, we define the over-approximated transition system on which we run our algorithm. We present a generic scheme for deciding reachability of upward closed sets in Section 6, and we show how to instantiate it on our model in Section 7. In Section 9, we report our experimental results on several tree protocols. Section 10 concludes the paper and gives direction for future works. Some proofs as well as the details of the case studies can be found in [1].

\section{Preliminaries}

In this section, we give some basic definitions and notations needed in the rest of the paper. To simplify the presentation, we will only consider binary trees in this paper. However, all the concepts and algorithms can be extended in a straightforward manner in order to deal with trees of higher ranks.

For a set $X$, we use $X^{*}$ to denote the set of words over $X$. We let $\varepsilon$ denote the empty word and use $x \bullet x^{\prime}$ to denote the concatenation of two words $x, x^{\prime} \in X^{*}$. We extend the concatenation operation to sets of words $D \subseteq X^{*}$ by $x \bullet D:=$ $\left\{x \bullet x^{\prime} \mid x^{\prime} \in D\right\}$. Given two words $x, x^{\prime} \in X^{*}$, we use $x \leq x^{\prime}$ to denote that $x$ is a prefix of $x^{\prime}$; and use $x<x^{\prime}$ to denote that $x \leq x^{\prime}$ and $x \neq x^{\prime}$. In case $x \leq x^{\prime}$, we use $x^{\prime}-x$ to denote the word $x^{\prime \prime}$ where $x \bullet x^{\prime \prime}=x^{\prime}$.

Binary Trees. A (binary) tree structure $N$ is a finite set of words over $\{0,1\}$ which is closed under the prefix relation, i.e. $n \in N$ and $n^{\prime} \leq n$ imply $n^{\prime} \in N$. In the rest of the paper, we fix a finite set of symbols $\Sigma$ and we use $b$ as a variable ranging over $\{0,1\}$.

A binary tree (tree for short) $T$ over the alphabet $\Sigma$ is a tuple $(N, \lambda)$ where $N$ is a tree structure and $\lambda$ is a mapping from $N$ to $\Sigma$. Each element of $N$ is called 
a node of $T$. We say that a node $n^{\prime}$ is the parent of the node $n$ iff $n^{\prime} \bullet b=n$ for some $b$. In such a case, $n$ is said to be $a$ child of $n^{\prime}$. A leaf in $T$ is a node which does not have any children; and the root of $T$ is the node $\varepsilon$. Given a node $n$, we define the descendants of $n$ by $\operatorname{Desc}(n):=\left\{n^{\prime} \in N \mid n<n^{\prime}\right\}$. We use Trees $(\Sigma)$ to denote the set of all trees over $\Sigma$.

Inclusions and Embeddings. Consider two trees $T=(N, \lambda)$ and $T^{\prime}=\left(N^{\prime}, \lambda^{\prime}\right)$ in Trees $(\Sigma)$.

An inclusion of $T$ in $T^{\prime}$ is an injection $f: N \rightarrow N^{\prime}$ such that for any $n \in N$ :

$-n \bullet b \in N \Longrightarrow f(n) \bullet b=f(n \bullet b)$, and

$-\lambda(n)=\lambda^{\prime}(f(n))$.

We write $T \subseteq f T^{\prime}$ to denote that $f$ is an inclusion of $T$ in $T^{\prime}$, and write $T \subseteq T^{\prime}$ if $T \subseteq_{f} T^{\prime}$ for some inclusion $f$. Informally, if $T \subseteq T^{\prime}$ then $T^{\prime}$ contains a copy of $T$.

An embedding of $T$ in $T^{\prime}$ is an injection $f: N \rightarrow N^{\prime}$ such that for any $n \in N$ :

$-n \bullet b \in N \Longrightarrow f(n) \bullet b \leq f(n \bullet b)$, and

$-\lambda(n)=\lambda^{\prime}(f(n))$.

We use $T \preceq_{f} T^{\prime}$ to denote that $f$ is an embedding of $T$ in $T^{\prime}$, and write $T \preceq T^{\prime}$ if $T \preceq_{f} T^{\prime}$ for some embedding $f$. Observe that $\preceq$ is a weaker relation than $\subseteq$. The difference between the two relations is that an inclusion preserves the parent/child relation between nodes, while an embedding preserves a weaker relation, namely that of ascendant/descendant.

Operations on Trees. In this paragraph, we fix a tree $T=(N, \lambda) \in \operatorname{Trees}(\Sigma)$.

For a node $n \in N$, we use $T(n)$ to denote the subtree of $T$ rooted at $n$. Formally, we let $T(n)=\left(N^{\prime}, \lambda^{\prime}\right)$ where $N^{\prime}:=\left\{n^{\prime \prime}-n \mid n^{\prime \prime} \in N \wedge n \leq n^{\prime \prime}\right\}$; and for any $n^{\prime} \in N^{\prime}, \lambda^{\prime}\left(n^{\prime}\right):=\lambda\left(n \bullet n^{\prime}\right)$.
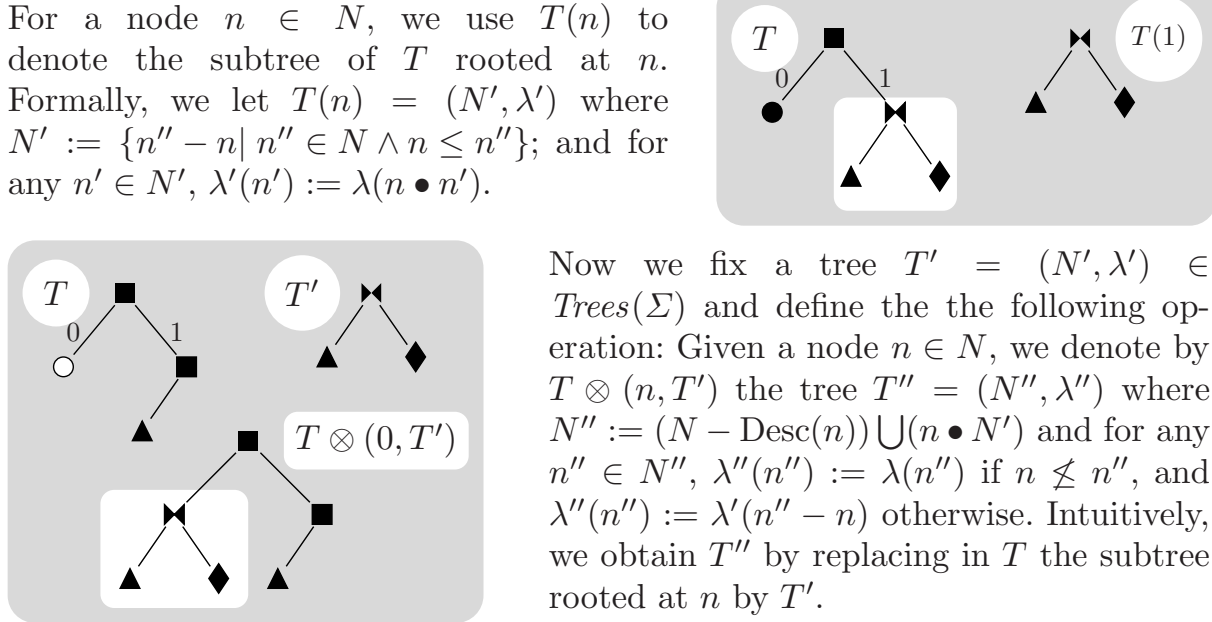

Now we fix a tree $T^{\prime}=\left(N^{\prime}, \lambda^{\prime}\right) \in$ Trees $(\Sigma)$ and define the the following operation: Given a node $n \in N$, we denote by $T \otimes\left(n, T^{\prime}\right)$ the tree $T^{\prime \prime}=\left(N^{\prime \prime}, \lambda^{\prime \prime}\right)$ where $N^{\prime \prime}:=(N-\operatorname{Desc}(n)) \bigcup\left(n \bullet N^{\prime}\right)$ and for any $n^{\prime \prime} \in N^{\prime \prime}, \lambda^{\prime \prime}\left(n^{\prime \prime}\right):=\lambda\left(n^{\prime \prime}\right)$ if $n \not n^{\prime \prime}$, and $\lambda^{\prime \prime}\left(n^{\prime \prime}\right):=\lambda^{\prime}\left(n^{\prime \prime}-n\right)$ otherwise. Intuitively, we obtain $T^{\prime \prime}$ by replacing in $T$ the subtree rooted at $n$ by $T^{\prime}$.

Consider a (partial) function $f: N \rightarrow N^{\prime}$. We define the renaming of $T^{\prime}$ with respect to $f$ and $T$, denoted by $T^{\prime} \odot_{f} T$, to be the tree $T^{\prime \prime}=\left(N^{\prime}, \lambda^{\prime \prime}\right)$ where for any $n^{\prime} \in N^{\prime}, \lambda^{\prime \prime}\left(n^{\prime}\right)=\lambda^{\prime}\left(n^{\prime}\right)$ if $n^{\prime} \notin \operatorname{Img}(f)$, and $\lambda^{\prime \prime}\left(n^{\prime}\right)=\lambda\left(f^{-1}\left(n^{\prime}\right)\right)$ otherwise. 


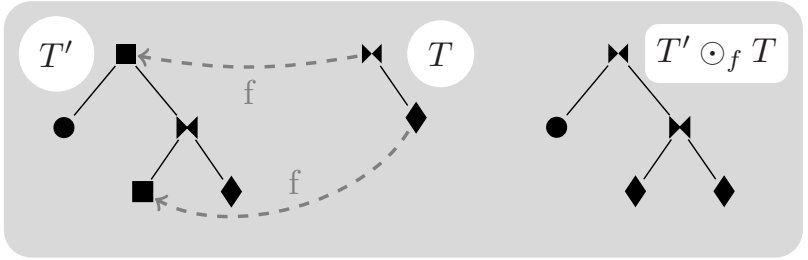

\section{Parameterized Tree Systems}

A parameterized tree system consists of an arbitrary (but finite) number of identical processes, arranged in a (binary) tree topology. Each process is a finite-state automaton. The transitions of the automaton are conditioned by the current local state and possibly the local states of other processes (parent, children, etc). A transition may change the states of all processes involved in the condition. A parameterized tree system induces an infinite family of finite-state systems, namely one for each size and each structure of the tree. The aim is to verify correctness of the systems for the whole family regardless of the number of processes in the system or the particular form of the tree.

Formally, a parameterized tree system $\mathcal{P}$ is a tuple $(Q, R)$ where $Q$ is a finite set of local states, and $R \subseteq \operatorname{Trees}(Q \times Q)$ is a finite set of trees called rewrite rules. For each rule $r=(N, \lambda) \in R$, we associate two special trees in $\operatorname{Trees}(Q)$ called left and right trees of $r$, and denoted respectively by $l h s(r)$ and $r h s(r)$. We define $\operatorname{lh} s(r):=(N, \operatorname{lh} s(\lambda))$ and $\operatorname{rhs}(r):=(N$, rhs $(\lambda))$, where $\operatorname{lhs}(\lambda)$ and $\operatorname{rhs}(\lambda)$ are obtained from $\lambda$ by projecting on the first and the second component of $Q \times Q$. More precisely, for any node $n \in N$, if $\lambda(n)=\left(q, q^{\prime}\right)$ then $\operatorname{lh} s(\lambda)(n):=q$ and $\operatorname{rhs}(\lambda)(n):=q^{\prime}$.

Example 1. We consider the percolate protocol where the set of states $Q$ is defined by $\left\{q_{0}, q_{1}, q_{u}\right\}$ and the transition rules $R=\left\{r_{1}, r_{2}, r_{3}, r_{4}\right\}$ are as depicted in Figure 2. The protocol evaluates the disjunction of the values in the leaves up to the root.
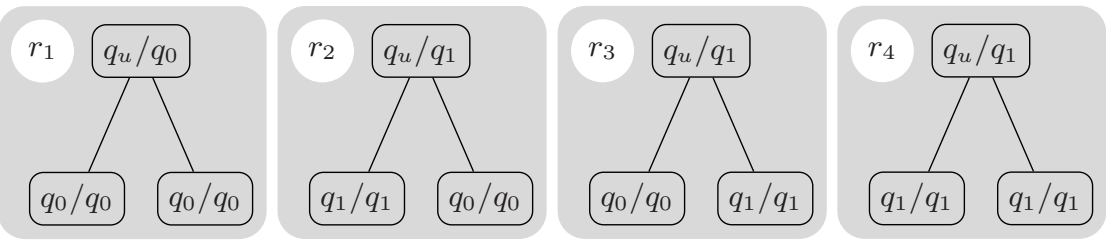

Fig. 2. The transition rules of the percolate protocol 


\section{Operational Semantics}

The operational semantics of a parameterized tree system can be captured by a transition system. In this section, we first describe the induced transition system. Then we introduce the coverability problem.

Transition System. A transition system $\mathcal{T}$ is a pair $(C, \Longrightarrow)$, where $C$ is an (infinite) set of configurations and $\Longrightarrow$ is a binary relation on $C$. We use $\stackrel{*}{\Longrightarrow}$ to denote the reflexive transitive closure of $\Longrightarrow$. Given an ordering $\unlhd$ on $C$, we say that $\mathcal{T}$ is monotonic with respect to $\unlhd$ if the following holds: For any configurations $c_{1}, c_{2}, c_{3} \in C$ with $c_{1} \Longrightarrow c_{3}$ and $c_{1} \unlhd c_{2}$, there is a configuration $c_{4} \in C$ such that $c_{2} \Longrightarrow c_{4}$ and $c_{3} \unlhd c_{4}$. We will consider several transition systems in this paper.

First, a parameterized system $\mathcal{P}=(Q, R)$ induces a transition system $\mathcal{T}(\mathcal{P})=$ $(C, \longrightarrow)$ where $C=\operatorname{Trees}(Q)$. Intuitively, a configuration $c=(N, \lambda) \in C$ represents an instance of the system with $|N|$ processes. These processes are arranged according to the tree structure $N$ and their current local states are given by $\lambda$. More precisely, each node $n \in N$ represents a process in the state $\lambda(n)$.

Next, we define the transition relation $\longrightarrow$ on the set of configurations as follows. Let $r \in R$ be a rewrite rule. Consider two configurations $c_{1}$ and $c_{2}$. We write $c_{1} \stackrel{r}{\longrightarrow} c_{2}$ to denote that there is an $f$ such that the following conditions hold: (i) $l h s(r) \subseteq_{f} c_{1}$, and (ii) $c_{2}=c_{1} \odot_{f} r h s(r)$. Intuitively, $c_{2}$ can be derived from $c_{1}$ by changing the labels of all the nodes in $\operatorname{Img}(f)$ according to the labeling function of $r h s(r)$. Below, we give informal explanations of the conditions. First, in condition (i), we identify the "active processes" (those which participate in the transition) by the inclusion $f(\operatorname{Img}(f))$. Implicitly, we interpret $l h s(r)$ as a guard and therefore require, through condition (i), that the configuration $c_{1}$ contains a tree which is a copy of the left hand side of the rule. Then, in condition (ii), we interpret $r h s(r)$ as an operation and require that, in $c_{2}$, the processes in $\operatorname{Img}(f)$ (the active ones) should all change state according to $r h s(r)$. Observe that the local states of the "passive processes", i.e. those not participating in the transition, should remain unchanged through the transition, and also that the transition does not change the structure of the tree ${ }^{1}$ (see Figure 3).

We use $c \longrightarrow c^{\prime}$ to denote that $c \stackrel{r}{\longrightarrow} c^{\prime}$ for some rule $r \in R$.

Safety Properties. In order to analyze safety properties, we study the coverability problem defined below. For a parameterized tree system $\mathcal{P}=(Q, R)$, we assume that we are given a set of initial configurations Init, each of which characterizes a possible state of the system prior to starting the execution.

We recall the definition of the relation $\preceq$ defined in Section 2. A set of configurations $D \subseteq C$ is said to be upward closed (with respect to $\preceq$ ) if $c \in D$ and $c \preceq c^{\prime}$ implies $c^{\prime} \in D$. For sets of configurations $D, D^{\prime} \subseteq C$ we use $D \longrightarrow D^{\prime}$

${ }^{1}$ In fact, our method can also cope with non-structure preserving rules, such dynamic creation and deletion of processes. However, for simplicity of presentation, we choose not to do so. 


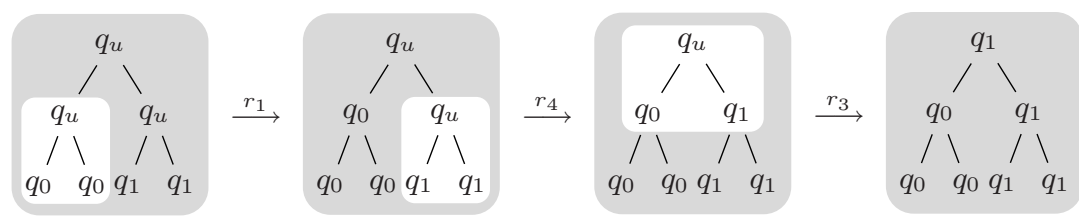

Fig. 3. A possible run of the percolate protocol. We highlight in white the zone where the rule applies (see Example 1).

to denote that there are $c \in D$ and $c^{\prime} \in D^{\prime}$ with $c \longrightarrow c^{\prime}$. The coverability problem for parameterized tree systems is defined as follows:

\section{PAR-TREE-COV}

\section{Instance}

- A parameterized tree system $\mathcal{P}=(Q, R)$.

- An upward closed set $F$ of configurations.

Question Init $\stackrel{*}{\longrightarrow}$ F ?

It can be shown, using standard techniques (see $[20,15]$ ), that checking safety properties (expressed as regular languages) can be translated into instances of the coverability problem. Therefore, checking safety properties amounts to solving PAR-TREE-COV (i.e. to the reachability of upward closed sets).

\section{Approximation}

In this section, we introduce an over-approximation of the transition relation of a parameterized tree system.

In Section 4, we mentioned that each parameterized tree system $\mathcal{P}=(Q, R)$ induces a transition system $\mathcal{T}(\mathcal{P})=(C, \longrightarrow)$. A parameterized tree system $\mathcal{P}$ also induces an approximate transition system $\mathcal{A}(\mathcal{P})=(C, \sim)$, where the set $C$ of configurations is identical to the one in $\mathcal{T}(\mathcal{P})$ and the transition relation $\leadsto$ is defined below.

First, we define a special operation on trees needed in order to describe the semantics of $\leadsto$.

Tree Subtraction . In this paragraph, we fix two trees $T=(N, \lambda), T^{\prime}=\left(N^{\prime}, \lambda^{\prime}\right) \in$ Trees $(\Sigma)$ such that $T^{\prime} \preceq_{f} T$ for some embedding $f$. We define $T \ominus_{f} T^{\prime}$ to be the tree $T^{\prime \prime}$ obtained from $T$ by performing a sequence of operations described below. First, we enumerate the nodes of $T^{\prime}$ in a bottom-up fashion. Formally, let $\left\{n_{i}\right\}_{1 \leq i \leq\left|N^{\prime}\right|}$ be an enumeration of the set $N^{\prime}$ of nodes in $T^{\prime}$ such that for any $i, j: 1 \leq i \neq j \leq\left|N^{\prime}\right|, n_{i}<n_{j}$ implies that $j<i$. In other words, if $n_{j}$ is a descendant of $n_{i}$ in $T^{\prime}$, then $n_{j}$ occurs earlier than $n_{i}$ in the enumeration. Based on the enumeration, we define a sequence of trees $\left\{T_{i}\right\}_{1 \leq i \leq\left|N^{\prime}\right|-1}$ as follows. We let $T_{1}:=T$. For any $i: 1 \leq i \leq\left|N^{\prime}\right|-2$, we denote by $n_{i}^{p}$ the parent of $n_{i}$, i.e. $n_{i}^{p} \bullet b=n_{i}$ for some $b$; and we define 


$$
T_{i+1}:=T_{i} \otimes\left(f\left(n_{i}^{p}\right) \bullet b, T\left(f\left(n_{i}\right)\right)\right) .
$$

Finally, we let $T^{\prime \prime}:=T_{\left|N^{\prime}\right|-1}$. In other words, we go through the nodes of $T^{\prime}$ one by one in a bottom-up manner. For each node $n_{i}$ and its parent $n_{i}^{p}$ in $T^{\prime}$ (say $n_{i}^{p} \bullet b=n_{i}$ for some $b$ ), we consider their images $f\left(n_{i}^{p}\right)$ and $f\left(n_{i}\right)$ in $T$. We replace the subtree rooted in the child of the image $f\left(n_{i}^{p}\right) \bullet b$ by the one rooted in the image $f\left(n_{i}\right)$ (see Figure 4). Notice that the resulting tree $T^{\prime \prime}$ and the trees $T^{\prime}, T$ are related by $T^{\prime} \subseteq T^{\prime \prime} \preceq T$. In the sequel, we denote by $\widehat{f}$ the inclusion of $T^{\prime}$ in $T^{\prime \prime}$ such that $\widehat{f}(\varepsilon)=\bar{f}(\varepsilon)$ (such a function exists and is unique by the definition above).
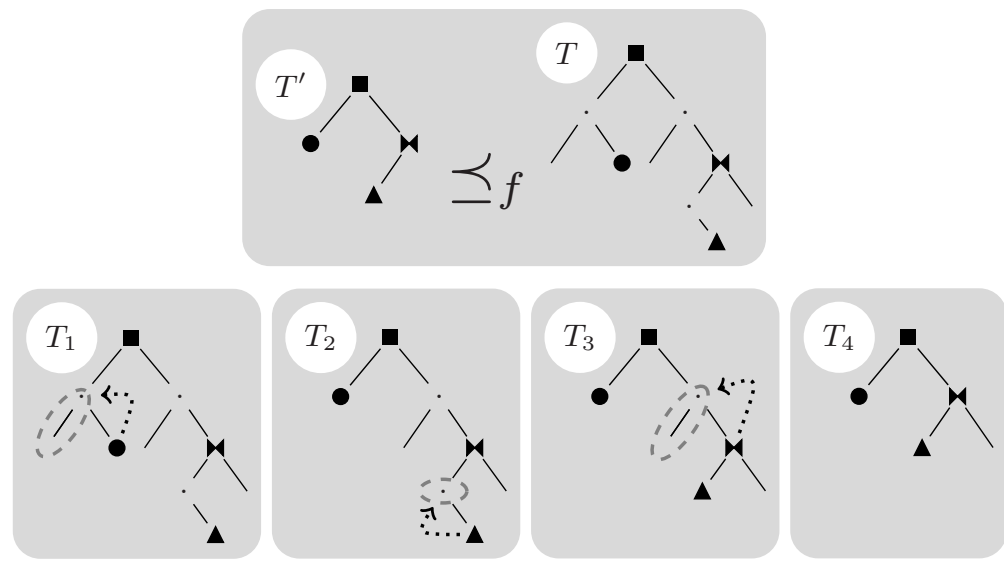

Fig. 4. In the first row, we give an example of two trees $T, T^{\prime}$ satisfying $T^{\prime} \preceq_{f} T$ for some embedding $f$. In the second row, we give the sequence of trees used in the definition of $T \ominus_{f} T^{\prime}$. In each of the trees, the arrow shows where subtrees are re-rooted, while the nodes surrounded by a dashed line are those which are removed.

The Approximate Transition Relation. Consider two configurations $c_{1}, c_{2}$ and a rule $r \in R$. We write $c_{1} \stackrel{r}{\sim} c_{2}$ to denote that there is an $f$ such that (i) lhs $(r) \preceq_{f} c_{1}$, and (ii) $c_{2}=\left(c_{1} \ominus_{f} \operatorname{lhs}(r)\right) \odot_{\widehat{f}} r h s(r)$. Intuitively, starting from $c_{1}$ and an embedding $f$ of $l h s(r)$ in $c_{1}$, we first remove all nodes in $c_{1}$ such that $\operatorname{lh} s(r)$ is included in the resulting configuration. This is done by taking lhs $(r) \ominus_{f} c_{1}$ and the inclusion $\widehat{f}$. Then we apply the rule $r$ and obtain $c_{2}$ from $\operatorname{lhs}(r) \ominus_{f} c_{1}$ in a similar manner to how it is described in the previous section, i.e. by renaming the labels of the nodes in $\operatorname{Img}(\widehat{f})$ according to $r h s(r)$ (see Figure 5 ). We use $c_{1} \leadsto 1 c_{2}$ if $c_{1} \stackrel{r}{\sim} c_{2}$ for some $r \in R$.

Observe that the relation $\leadsto$ is an over-approximation of the transition relation defined in the previous section (i.e. $\leadsto \supseteq \longrightarrow$ ) by the following argument. 

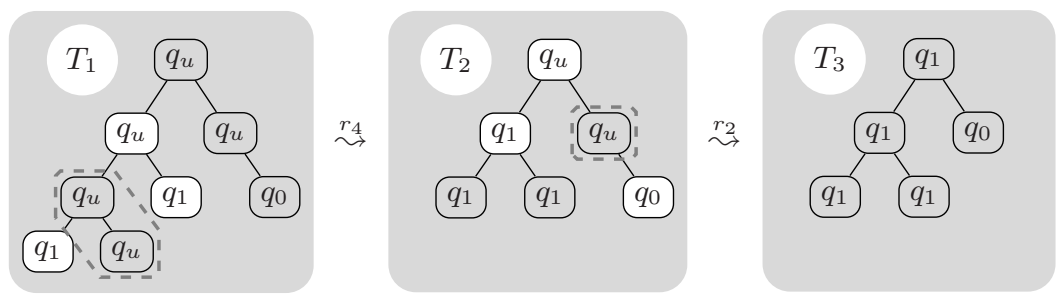

Fig. 5. A possible run of the approximate transition system induced by the percolate protocol (see Example 1). The nodes with a white background represent those where the rule will apply while the dashed lines surround the nodes which are removed.

Consider two configurations $c_{1}, c_{2} \in C$ with $c_{1} \longrightarrow c_{2}$. By definition, this implies the existence of a rule $r \in R$ and an inclusion $f$ of $l h s(r)$ in $c_{1}$ such that $c_{2}=c_{1} \odot_{f} r h s(r)$. Observe that, by definition of the $\ominus$ operation, since $f$ is an inclusion it follows that $c_{1} \ominus_{f} \operatorname{lh} s(r)=c_{1}$ and $\widehat{f}=f$. Therefore, we obtain $c_{2}=c_{1} \odot_{f} r h s(r)=\left(c_{1} \ominus_{f} \operatorname{lhs}(r)\right) \odot_{\widehat{f}} r h s(r)$, and as a consequence, $c_{1} \stackrel{r}{\sim} c_{2}$.

We are now ready to state a key property of the approximated transition system.

Lemma 1. The approximate transition system $(C, \leadsto)$ is monotonic with respect to $\preceq$.

We define the coverability problem for the approximate system as follows.

\section{APRX-PAR-TREE-COV \\ Instance}

- A parameterized tree system $\mathcal{P}=(Q, R)$

- An upward closed set $F$ of configurations.

Question Init $\stackrel{*}{\sim} F$ ?

Since $\longrightarrow \subseteq \sim$, a negative answer to APRX-PAR-TREE-COV implies a negative answer to PAR-TREE-COV.

\section{Scheme}

In this section, we recall a generic scheme from [2] for performing symbolic backward reachability analysis. The scheme in question is based on symbolic representations of infinite sets of configurations called constraints. Throughout this section, we fix a transition system $\mathcal{T}=(C, \Longrightarrow)$ and a set Init $\subseteq C$ of initial configurations.

Constraint Systems. A constraint system $\Psi$ relative to the transition system $\mathcal{T}$ is a set whose elements are called constraints and can be finitely encoded, such that there is a function $\llbracket \cdot \rrbracket: \Psi \rightarrow 2^{C}$. For a finite set $\Phi$ of constraints, we let $\llbracket \Phi \rrbracket=\bigcup_{\phi \in \Phi} \llbracket \phi \rrbracket$. We say that a set $D \subseteq C$ is computable or representable (in the 
constraint system $\Psi$ ) if it is possible to compute a finite set of constraints $\Phi \subseteq \Psi$ such that $D=\llbracket \Phi \rrbracket$.

We define an entailment relation $\sqsubseteq$ on constraints, where $\phi_{1} \sqsubseteq \phi_{2}$ iff $\llbracket \phi_{2} \rrbracket \subseteq$ $\llbracket \phi_{1} \rrbracket$. For sets $\Phi_{1}, \Phi_{2}$ of constraints, abusing notation, we let $\Phi_{1} \sqsubseteq \Phi_{2}$ denote that for each $\phi_{2} \in \Phi_{2}$ there is a $\phi_{1} \in \Phi_{1}$ with $\phi_{1} \sqsubseteq \phi_{2}$. Notice that $\Phi_{1} \sqsubseteq \Phi_{2}$ implies that $\llbracket \Phi_{2} \rrbracket \subseteq \llbracket \Phi_{1} \rrbracket$.

For a constraint $\phi$, we let $\operatorname{Pre}(\phi)$ be the set of constraints, such that $\llbracket \operatorname{Pre}(\phi) \rrbracket=$ $\left\{c \mid \exists c^{\prime} \in \llbracket \phi \rrbracket . c \Longrightarrow c^{\prime}\right\}$. In other words, Pre $(\phi)$ characterizes the set of configurations from which we can reach a configuration in $\phi$ through the application of a single rewrite rule. Such a set does not necessarily exist, nevertheless, for our class of systems, we will show that such a set always exists and is in fact computable. For a set $\Phi$ of constraints, we let $\operatorname{Pre}(\Phi)=\bigcup_{\phi \in \Phi} \operatorname{Pre}(\phi)$.

Symbolic Backward Reachability . We present a scheme for a symbolic algo-

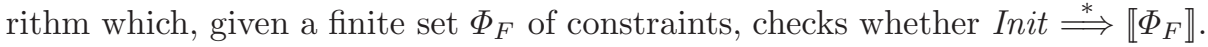

In the scheme, we perform a backward reachability analysis, generating a sequence $\left\{\Phi_{i}\right\}_{i \in \mathbb{N}}: \Phi_{0} \sqsupseteq \Phi_{1} \sqsupseteq \Phi_{2} \sqsupseteq \cdots$ of finite sets of constraints such that $\Phi_{0}=\Phi_{F}$, and $\Phi_{i+1}=\Phi_{i} \cup \operatorname{Pre}\left(\Phi_{i}\right)$. Since $\llbracket \Phi_{0} \rrbracket \subseteq \llbracket \Phi_{1} \rrbracket \subseteq \llbracket \Phi_{2} \rrbracket \subseteq \cdots$, the procedure terminates when we reach a point $j$ where $\Phi_{j} \sqsubseteq \Phi_{j+1}$. Consequently, $\Phi_{j}$ characterizes the set of all predecessors of $\llbracket \Phi_{F} \rrbracket$. This means that Init $\stackrel{*}{\Longrightarrow}$ $\llbracket \Phi_{F} \rrbracket$ iff Init $\cap \llbracket \Phi_{j} \rrbracket \neq \emptyset$.

Observe that, in order to implement the scheme (i.e. transform it into an algorithm), we need to be able to (i) compute Pre; (ii) check for entailment between constraints; and (iii) check for emptiness of Init $\cap \llbracket \phi \rrbracket$ for any constraint $\phi$. A constraint system satisfying these three conditions is said to be effective. Moreover, in [2], it is shown that termination is guaranteed in case the constraint system is well quasi-ordered (WQO) with respect to $\sqsubseteq$, i.e. for each infinite sequence $\phi_{0}, \phi_{1}, \phi_{2}, \ldots$ of constraints, there are $i<j$ with $\phi_{i} \sqsubseteq \phi_{j}$.

\section{Algorithm}

In this section, we instantiate the scheme of Section 6 to derive an algorithm for solving APRX-PAR-TREE-COV. We do that by introducing an effective and well quasi-ordered constraint system.

Throughout this section, we assume a parameterized tree system $\mathcal{P}=(Q, R)$ and the induced approximate transition system $\mathcal{A}(\mathcal{P})=(C, \leadsto)$. We define a constraint to be a tree in Trees $(Q)$. Although we use the same syntax as for configurations, constraints are interpreted differently. More precisely, given a constraint $\phi$, we let $\llbracket \phi \rrbracket=\{c \in C \mid \phi \preceq c\}$.

An aspect of our constraint system is that each constraint characterizes a set of configurations which is upward closed with respect to $\preceq$. Conversely (by Higman's Lemma [16]), any upward closed set $F$ of configurations can be characterized as $\llbracket \Phi_{F} \rrbracket$ where $\Phi_{F}$ is a finite set of constraints. In this manner, APRX-PAR-TREE-COV is reduced to checking the reachability of a finite set of constraints. 
Below we show effectiveness and well quasi-ordering of our constraint system, meaning that we obtain an algorithm for solving APRX-PAR-TREE-COV. First, observe that the entailment relation can be computed in a straightforward manner since for any constraints $\phi, \phi^{\prime}$, we have $\phi \sqsubseteq \phi^{\prime}$ iff $\phi \preceq \phi^{\prime}$.

In order to check the initial condition, we rely on previous works on regular tree languages [13] and provide a sufficient condition on Init which guarantees effectiveness of Init $\cap \llbracket \phi \rrbracket=\emptyset$ for any constraint $\phi$. More precisely, we require that the set Init can be characterized by a regular tree language.

For the computation of Pre we rely on the following result.

Lemma 2. For any constraint $\phi$, the set of constraints Pre $(\phi)$ is computable and finite.

It was shown in [19] that the embedding relation on trees $\preceq$ is a well quasiorder (Kruskal's theorem). This combined with results in [2] guarantee termination of our scheme when instantiated on the constraints we have defined above.

\section{Case Studies}

In this section, we provide descriptions of two tree protocols we have analyzed using our method. For each protocol, we define the corresponding parameterized tree system model and we give the sets of unsafe $(F)$ and initial (Init) configurations.

\subsection{The Tree-Arbiter Protocol}

The protocol supervises the access to a shared resource of a set of processes arranged in a tree topology. The processes competing for the resource reside in the leaves.

A process in the protocol can be in state $i d l e(i)$, requesting $(r)$, token $(t)$ or below $(b)$. All the processes are initially in state $i$. A node is in state $b$ whenever it has a descendant in state $t$. When a leaf is in state $r$, the request is propagated upwards until it encounters a node which is aware of the presence of the token (i.e. a node in state $t$ or $b$ ). A node that has the token (in state $t$ ) can choose to pass it upwards or pass it downwards to a requesting child (node in state $r$ ).

We model the tree-arbiter protocol with a parameterized tree system $\mathcal{P}=$ $(Q, R)$ where $Q=\left\{q_{s}^{n} \mid s \in\{i, r, t, b\} \wedge n \in\{\right.$ leaf, inner, root $\left.\}\right\}$ and $R$ is as depicted in the figure below (figure 6). Observe that in the definition of $Q$, we use the scripts $s$ and $n$ to model respectively the state and the nature (leaf, inner or root) of the nodes. In the definition of the rules, we will drop the script(s) whenever we mean that it is arbitrary (it can take any value).

The rules to model this protocol are as follows: 2 rules to propagate the request upwards, 2 rules to propagate the token downwards, 2 rules to propagate the token upwards and one rule to initiate a request from a leaf.

The set of bad constraints $F$ is represented by trees where at least two processes (i.e. two leaves) obtain the token (i.e. in state $q_{t}^{\text {leaf }}$ ). 


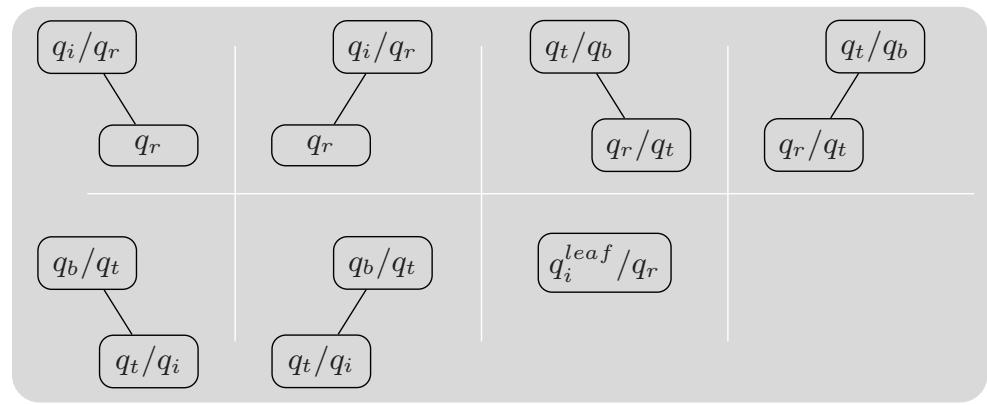

Fig. 6. The rewrite rules for the tree-arbiter protocol. We mention here that there are more rules in the model we have verified. For example, the rule in the top-left corner is represented in the concrete model by 2 rules, each of which corresponds to a particular combination of the natures of the parent and child nodes: For the parent there are 2 possibilities $\left(q_{i}^{\text {inner }} / q_{r}^{\text {inner }}\right.$ and $\left.q_{i}^{\text {root }} / q_{r}^{\text {root }}\right)$ while for the child, there are $2\left(q_{r}^{\text {inner }}\right.$ and $\left.q_{r}^{\text {leaf }}\right)$.

The set of initial configurations Init contains all trees where the leaf nodes are either idle or requesting, inner nodes are idle, and the root has the token.

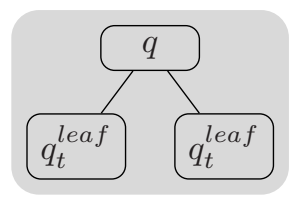

\subsection{The IEEE 1394 Tree Identification Protocol}

The 1394 High Performance serial bus [17] is used to transport digitized video and audio signals within a network of multimedia systems and devices.

The tree identification protocol is used in one of the phases implementing the IEEE 1394 protocol. More precisely, it is run after a bus reset in the network and leads to the election of a unique leader node.

In this section, we consider a version working on tree topologies. Furthermore, we assume that (i) each inner node is connected to 3 neighbors, (ii) the root is connected to 2 neighbors, and (ii) communication is atomic.

Initially, all nodes are in state undefined $(u)$. We identify two steps in the protocol depending on the number $n$ of neighbors which are still in state $u$. If $n>1$, the node waits for ("be my parent") requests from its neighbors. If $n=1$, the node sends a request to the remaining neighbor in state $u$. Observe that we implicitly assume that the leaf nodes are the first to communicate with their neighbors.

Formally, we derive a parameterized tree system model $\mathcal{P}=(Q, R)$ as follows. We define the set of states by $Q=\left\{q_{s}^{n} \mid s \in\{u, c, l\} \wedge n \in\{\right.$ leaf, inner, root $\left.\}\right\}$ where the scripts $s$ and $n$ describe respectively the state and the nature of the node. In the definition of the state $(s)$, the letters $u, c$ and $l$ stand respectively for undefined, child and leader. In a similar manner to the previous section, we 
drop the script(s) whenever we mean that it can take any value (see caption of Figure 6).

The rewrite rules $R$ are described below.

- The leaves initiate the communications:
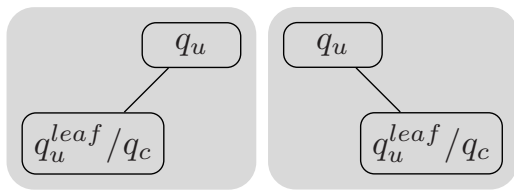

- The inner nodes become children or wait for requests:
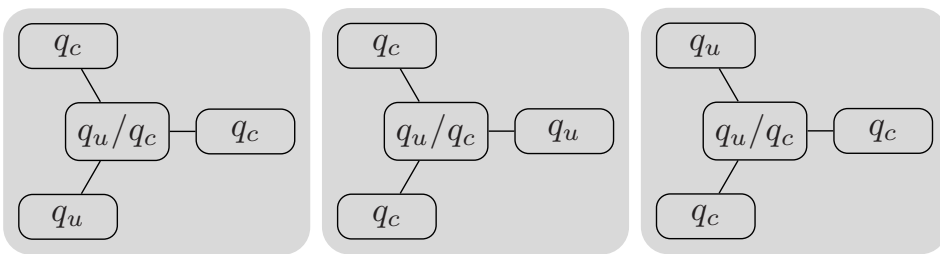

- The leader is chosen:
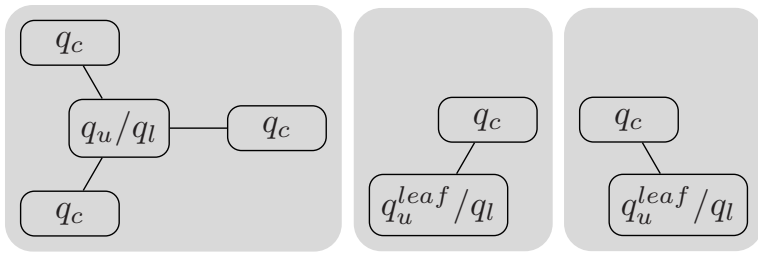

The set of initial configurations Init is represented by trees where all nodes are in state undefined, and the set of bad constraints $F$ is represented by trees where at least 2 leaders are elected.
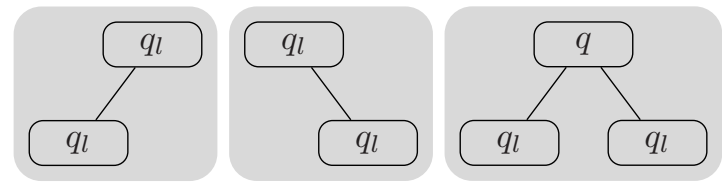

\section{$9 \quad$ Experiments}

We have implemented a prototype tool in $\mathrm{C}++$ and run it on several models of protocol with tree-like topologies. The experiments have been performed on a dual Opteron $2.8 \mathrm{GHz}$, with $8 \mathrm{~GB}$ of RAM memory and the results are reported in Table 1.

For each example, we give the number of iterations performed by the reachability algorithm, the largest number of constraints maintained at the end of the execution, the time and total memory consumption. Full details of the examples can be found in [1]. 
Table 1. Experimental Results

\begin{tabular}{|l|c|c|c|c|}
\hline Protocol & Time & \# iterations & \# constraints & Memory \\
\hline \hline Token & $1 \mathrm{~s}$ & 1 & 3 & $<1 \mathrm{MB}$ \\
Two way token & $1 \mathrm{~s}$ & 1 & 3 & $<1 \mathrm{MB}$ \\
Percolate & $1 \mathrm{~s}$ & 1 & 2 & $<1 \mathrm{MB}$ \\
Leader & $1 \mathrm{~s}$ & 4 & 41 & $63 \mathrm{MB}$ \\
Tree Arbiter & $37 \mathrm{~s}$ & 12 & 1173 & $70 \mathrm{MB}$ \\
IEEE 1394 & $1 \mathrm{~h} 15 \mathrm{~m} 25 \mathrm{~s}$ & 17 & 4145 & $137 \mathrm{MB}$ \\
\hline \hline
\end{tabular}

\section{Conclusions and Future Work}

We have presented a method for verification of tree parameterized systems where the components are organized in a tree. We derive an over-approximation of the transition relation which allows the use of symbolic reachability analysis defined on upward closed sets of trees (configurations). This technique has been implemented and successfully tested on a number of tree-based protocols.

It would be interesting to see if one can extend our method to other classes of architectures such as unordered trees, DAGs, and more general classes of graphs. In a similar manner to the case of words [3] we intend to consider tree systems where the individual processes may contain unbounded variables. This would allow to analyze algorithms for manipulation of heaps, (balanced) binary trees, etc. Finally, we intend to extend our framework to check for liveness properties on tree-like architecture systems (as done for words in [6]).

\section{References}

1. Abdulla, P., Henda, N.B., Delzanno, G., Haziza, F., Rezine, A.: Parameterized tree systems. Technical Report 2008-010, Dept. of Information Technology, Uppsala University, Sweden (March 2008)

2. Abdulla, P.A., Čerāns, K., Jonsson, B., Tsay, Y.-K.: General decidability theorems for infinite-state systems. In: Proc. LICS 1996, 11th IEEE Int. Symp. on Logic in Computer Science, pp. 313-321 (1996)

3. Abdulla, P.A., Delzanno, G., Rezine, A.: Parameterized verification of infinite-state processes with global conditions. In: Damm, W., Hermanns, H. (eds.) CAV 2007. LNCS, vol. 4590, pp. 145-157. Springer, Heidelberg (2007)

4. Abdulla, P.A., Jonsson, B., Mahata, P., d'Orso, J.: Regular tree model checking. In: Brinksma, E., Larsen, K.G. (eds.) CAV 2002. LNCS, vol. 2404, Springer, Heidelberg (2002)

5. Abdulla, P.A., Jonsson, B., Nilsson, M., d'Orso, J.: Regular model checking made simple and efficient. In: Brim, L., Jančar, P., Křetínský, M., Kucera, A. (eds.) CONCUR 2002. LNCS, vol. 2421, pp. 116-130. Springer, Heidelberg (2002)

6. Abdulla, P.A., Jonsson, B., Nilsson, M., d'Orso, J., Saksena, M.: Regular model checking for s1s + ltl. In: Alur, R., Peled, D.A. (eds.) CAV 2004. LNCS, vol. 3114, pp. 348-360. Springer, Heidelberg (2004) 
7. Abdulla, P.A., Legay, A., d'Orso, J., Rezine, A.: Tree regular model checking: A simulation-based approach. The Journal of Logic and Algebraic Programming 69(12), 93-121 (2006)

8. Alur, R., Brayton, R.K., Henzinger, T.A., Qadeer, S., Rajamani, S.K.: Partialorder reduction in symbolic state space exploration. In: Grumberg, O. (ed.) CAV 1997. LNCS, vol. 1254, pp. 340-351. Springer, Heidelberg (1997)

9. Boigelot, B., Legay, A., Wolper, P.: Iterating transducers in the large. In: Hunt Jr., W.A., Somenzi, F. (eds.) CAV 2003. LNCS, vol. 2725, pp. 223-235. Springer, Heidelberg (2003)

10. Bouajjani, A., Habermehl, P., Vojnar, T.: Abstract regular model checking. In: Alur, R., Peled, D.A. (eds.) CAV 2004. LNCS, vol. 3114, pp. 372-386. Springer, Heidelberg (2004)

11. Bouajjani, A., Jonsson, B., Nilsson, M., Touili, T.: Regular model checking. In: Emerson, E.A., Sistla, A.P. (eds.) CAV 2000. LNCS, vol. 1855, pp. 403-418. Springer, Heidelberg (2000)

12. Bouajjani, A., Touili, T.: Extrapolating Tree Transformations. In: Brinksma, E., Larsen, K.G. (eds.) CAV 2002. LNCS, vol. 2404, Springer, Heidelberg (2002)

13. Comon, H., Dauchet, M., Gilleron, R., Jacquemard, F., Lugiez, D., Tison, S., Tommasi, M.: Tree Automata Techniques and Applications (October 1999)

14. Dams, D., Lakhnech, Y., Steffen, M.: Iterating transducers. In: Berry, G., Comon, H., Finkel, A. (eds.) CAV 2001. LNCS, vol. 2102, Springer, Heidelberg (2001)

15. Godefroid, P., Wolper, P.: Using partial orders for the efficient verification of deadlock freedom and safety properties. Formal Methods in System Design 2(2), 149164 (1993)

16. Higman, G.: Ordering by divisibility in abstract algebras. Proc. London Math. Soc (3) 2(7), 326-336 (1952)

17. IEEE Computer Society. IEEE standard for a high performance serial bus. Std 1394-1995 (August 1996)

18. Kesten, Y., Maler, O., Marcus, M., Pnueli, A., Shahar, E.: Symbolic model checking with rich assertional languages. Theoretical Computer Science 256, 93-112 (2001)

19. Kruskal, J.: Well-quasi-ordering, the tree theorem, and Vazsonyi's conjecture. Transactions of the American Mathematical Society 95, 210-225 (1960)

20. Vardi, M.Y., Wolper, P.: An automata-theoretic approach to automatic program verification. In: Proc. LICS 1986, 1st IEEE Int. Symp. on Logic in Computer Science, June 1986, pp. 332-344 (1986) 